Proceedings of the 13th International Symposium UFPS, Vilnius, Lithuania 2007

\title{
Electrical Properties and Magnetoresistance of Nanogranular $\mathrm{SnO}_{2}$ Films
}

\author{
V.K. Ksenevich ${ }^{a, *}$, T.A. Dovzhenko ${ }^{a}$, V.A. Dorosinets ${ }^{a}$, \\ I.A. Bashmakov ${ }^{b}$, A.A. MElnikov ${ }^{c}$ AND A.D. WiecK ${ }^{c}$ \\ ${ }^{a}$ Department of Physics, Belarus State University \\ Nezalezhnasti av.4, 220030 Minsk, Belarus \\ ${ }^{b}$ Department of Chemistry, Belarus State University \\ Leningradskayja str. 14, 220030 Minsk, Belarus \\ ${ }^{c}$ Department of Physics and Astronomy, Bochum Ruhr-University \\ Universitaetstr., 150, 44780 Bochum, Germany
}

Magnetotransport properties of the nanogranular $\mathrm{SnO}_{2}$ films were invesigated. Non-linear current-voltage $(I-V)$ characteristics were observed at low temperatures. The temperature dependence of the resistance and non-ohmic $I-V$ curves can be well approximated by fluctuation-induced tunnelling model, indicating importance of the contacts barriers between $\mathrm{SnO}_{2}$ grains. Magnetoresistance was measured within temperature range 2$15.3 \mathrm{~K}$ and could be consistent with the variable-range hopping conduction mechanism due to existence of localized states on the surface of $\mathrm{SnO}_{2}$ grains.

PACS numbers: 73.43.Qt, 73.50.Fq

\section{Introduction}

$\mathrm{SnO}_{2}$ films and nanostructures are known as prospective materials for gas sensors [1], solar cells [2], photoconductors [3], lithium batteries [4] and transparent conductive coatings [5] due to their excellent electrical properties such as good electrical conductivity, high transparency in the visible region and high reflectivity for infrared radiation. The properties of $\mathrm{SnO}_{2}$ are mainly determined by doping, interfaces and defects. We report here magnetotransport properties of $\mathrm{SnO}_{2}$ nanogranular films.

\section{Experimental}

Samples were synthesized using combination of the method of thermoconversion of metal-polymer precursor in vacuum with gas-transport chemical reaction and successive heat-treatment procedure. The sizes of the $\mathrm{SnO}_{2}$ grains were in the

${ }^{*}$ corresponding author; e-mail: ksenevich@bsu.by 
range 50-100 $\mathrm{nm}$ according to scanning electron microscopy images. The transport properties of the nanogranular $\mathrm{SnO}_{2}$ films were measured using standard four-probe dc-technique in the temperature range 2-300 K. Electrical contacts were produced by thermal evaporation of $\mathrm{Al}$ on the top of the layers. The $I-V$ characteristics were measured at room temperature and in the low temperature range $(2-15.3 \mathrm{~K})$. The temperature dependences of the resistance $R(T)$ were measured in the range $4.2-300 \mathrm{~K}$. Magnetoresistance (MR) measurements were made in the magnetic fields up to $5 \mathrm{~T}$ in the temperature range $2-15.3 \mathrm{~K}$.

\section{Results and discussion}

At room temperature the $I-V$ curves were linear. In the low temperature range non-ohmic behavior appeared on $I-V$ curves and became essential at $T=2 \mathrm{~K}$. As shown in Fig. 1 by the fitted lines, non-linear $I-V$ curves are well described by the expression

$$
I=G_{0} V \exp \left(V / V_{0}\right),
$$

where parameter $G_{0}$ is the low-field conductance, $V_{0}$ is the scale parameter. Equation (1) was obtained by Kaiser et al. [6] by means of numerical calculations of fluctuation-induced tunnelling and thermal activation (extending the fluctuationinduced tunnelling model of Sheng [7] to cases of higher conductivity). This model was proposed for different disordered systems in which most of conduction electrons are delocalized and free to move over large (in atomic scale) distances. Therefore, the electrical conduction in these systems is dominated by electrical transport between large conducting regions rather than by hopping between localized states. Tunneling probability in these systems depends on temperature due to existence of thermally activated voltage fluctuations across the junction. It should be noted that the parameter $G_{0}$ shows a strong growth with temperature (more than 1 order of magnitude as the temperature was rised from 2 to $15.3 \mathrm{~K}$ ), while the parameter $V_{0}$ shows only a relatively small increase with temperature. This behavior is consistent with the fluctuation-induced tunnelling model $[6,7]$.

The $R(T)$ dependences show negative $\mathrm{d} R / \mathrm{d} T$ value in the whole investigated temperature range and also follow the thermal fluctuation-induced tunnelling model [7]:

$$
R=R_{0} \exp \left(T_{1} / T+T_{0}\right)
$$

where $T_{1}$ according to the model [7] denotes the temperature below which the conduction is dominated by the charge carrier tunnelling through the barrier and $T_{0}$ - the temperature above which fluctuation effects become significant. Fitting of $R(T)$ by Eq. (2) gave the values $T_{0}=94.6 \mathrm{~K}$ and $T_{1}=287.5 \mathrm{~K}$.

As the temperature was decreased below $40 \mathrm{~K}$, deviation from the law (2) was observed due to influence of other conductivity mechanism on the charge transport in the samples. One of the possible charge transport mechanism is variable-range hopping $(\mathrm{VRH})$ which can originate from the existence of the localized states on the surface of $\mathrm{SnO}_{2}$ grains. This assumption is confirmed by MR data. 


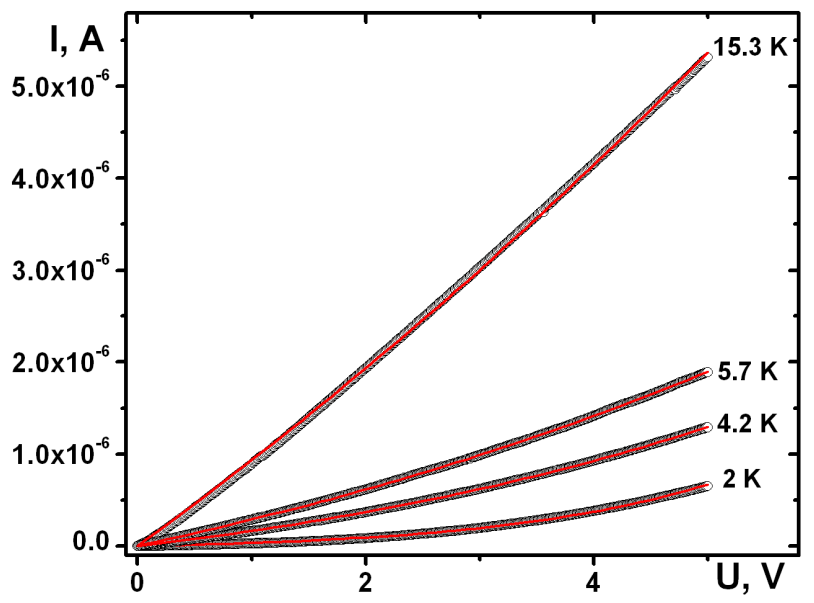

Fig. 1. $I-V$ characteristics of the $\mathrm{SnO}_{2}$ nanogranular films at low temperatures. The lines are fits to data by Eq. (1).

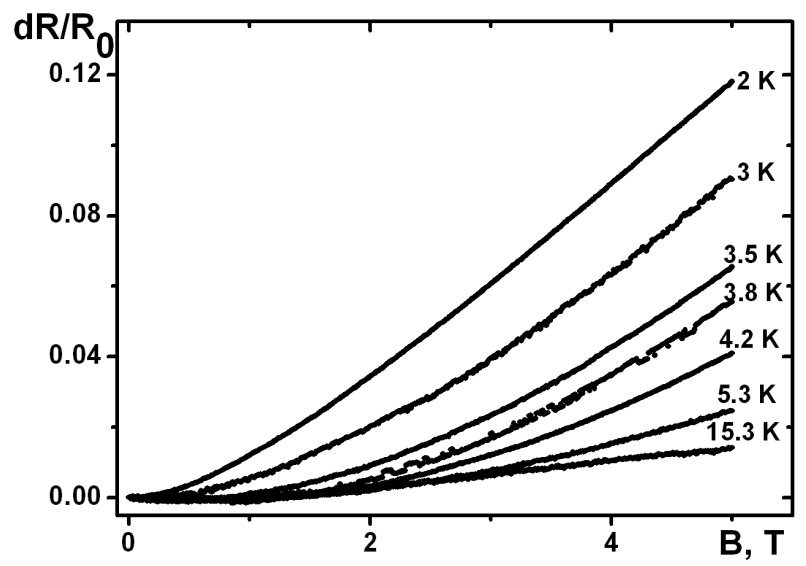

Fig. 2. The dependence of the normalized change of the resistance $\mathrm{d} R / R_{0}$ of the $\mathrm{SnO}_{2}$ nanogranular films on the magnetic field in the range $2-15.3 \mathrm{~K}$.

MR was positive and increased exponentially with the temperature as one can see in Fig. 2. This behavior is typical of VRH conduction [8]. The value of positive MR decreased as the temperature was raised. The small negative MR in the range of low magnetic fields was observed. The minimum position of negative MR shifted to higher fields as the temperature was raised. Such MR behavior can be interpreted by means of theory of negative MR in the strong localization (SL) regime due to influence of quantum interference effects on VRH conduction [9]. Thus, MR data could be consistent with the VRH conduction mechanism due to existence of localized states. 
In conclusion, electrical properties and magnetoresistance of $\mathrm{SnO}_{2}$ nanogranular films were investigated. Combinations of the fluctuation-induced tunnelling and VRH conduction determine the electrical and magnetotransport properties of the system. Contact potential barriers between $\mathrm{SnO}_{2}$ grains and localized states on their surfaces are believed to determine the charge transport properties of the structures.

\section{Acknowledgments}

The work was supported by the Foundation of Basic Research of Belarus (project No. FS05M-167). One of us (V.K.) would like to acknowledge DAAD for financial support and Prof. A.D. Wieck for hospitality.

\section{References}

[1] Y. Zhang, A. Kolmakov, S. Chretien, H. Metiu, M. Moskovits, Nano Lett. 4, 403 (2004).

[2] S. Ferrere, A. Zaban, B.A. Gsegg, J. Phys. Chem. B 101, 4490 (1997).

[3] S. Mathur, S. Barth, H. Shen, J. C. Pyun, U. Werner, Small 1, 713 (2005).

[4] J. Zhu, Zh. Lu, S.T. Aruna, D. Aurbach, A. Gedanken, Chem. Matter 12, 2557 (2000).

[5] K.L. Chopra, S. Major, D.K. Pandya, Thin Solid Films 102, 1 (1993).

[6] A.B. Kaiser, S.A. Rogers, Y.W. Park, Mol. Cryst. Liq. Cryst. 415, 115 (2004).

[7] P. Sheng, Phys. Rev. B 21, 2180 (1980).

[8] B.I. Shklovskii, A.L. Efros, Electronic Properties of Doped Semiconductors, Springer-Verlag, Berlin 1984.

[9] U. Sivan, O. Entin-Wohlman, Y. Imry, Phys. Rev. Lett. 60, 1566 (1988). 